\title{
Two case reports of 'locked spondyloptosis': the most severe traumatic cervical spondyloptosis with locked spinous process and vertebral arch into the spinal canal
}

\author{
Takeru Tsujimoto $\mathbb{1}^{1,2} \cdot$ Kota Suda $^{1} \cdot$ Satoko Matsumoto Harmon ${ }^{1} \cdot$ Miki Komatsu $^{1} \cdot$ Masahiko Takahata $^{2}$. \\ Norimasa Iwasaki ${ }^{2} \cdot$ Akio Minami $^{1}$
}

Received: 27 December 2019 / Revised: 6 February 2020 / Accepted: 6 February 2020

(c) International Spinal Cord Society 2020

\begin{abstract}
Introduction Traumatic cervical spondyloptosis, including compressive-extension stage 5 of Allen's classification of cervical spine injuries, is commonly observed; however, cases involving locked spinous process and vertebral arch into the spinal canal are extremely rare.

Case presentation We present two individuals with spondyloptosis of C7 with locked spinous process of C6 and the vertebral arch into the spinal canal. Closed reduction was unable to be performed due to rigid locking of the cervical spine in the first case, whereas preoperative closed reduction was achieved with mild traction in a prone position after general anaesthesia in the second case. These two individuals underwent spinal fusion via a posterior approach after open or closed reduction. Six months after surgery, both individuals exhibited significant neurological recovery and acquired a stable gait. Discussion To the best of our knowledge, this is the first report of traumatic 'locked spondyloptosis' of the spinous process and vertebral arch into the spinal canal. Although high-grade compressive-extension injuries are usually repaired using a combined anterior-posterior approach, repair is possible with a posterior approach alone with reliable anchors, such as pedicle screws or multiple lateral mass screws. Urgent open reduction may be required for locked spondyloptosis when closed reduction is invalid due to rigid locking of the cervical spine.
\end{abstract}

\section{Introduction}

Traumatic cervical spondyloptosis, in which the affected vertebral body is completely dislocated to the anterior side of the subjacent vertebral body, is the most severe form of cervical spine injury [1-4]. Traumatic cervical spondyloptosis often represents a type of compressive-extension stage (CES) 5 injury of Allen's classification of cervical spine injuries [5]. Although cases of CES 5 are common, there are no reports of traumatic cervical spondyloptosis with locked spinous process and vertebral arch into the spinal canal.

Takeru Tsujimoto

t.1105.tsujimoto@gmail.com

1 Department of Orthopaedic Surgery, Hokkaido Spinal Cord Injury Center, Hokkaido, Japan

2 Department of Orthopaedic Surgery, Faculty of Medicine and Graduate of Medicine, Hokkaido University, Hokkaido, Japan
We report two cases of the most severe type of traumatic cervical spondyloptosis, in which closed reduction may be impossible due to rigid dislocation and locking of the cervical spine. To the best of our knowledge, this is the first report of the traumatic 'locked spondyloptosis' of the spinous process and vertebral arch into the spinal canal.

\section{Case presentation}

\section{Case 1}

A 69-year-old man was involved in a motor vehicle collision, during which his head collided against the windscreen, resulting in extreme neck pain, paraplegia, and difficulty using his hands. The individual was transferred to our institute $\sim 6 \mathrm{~h}$ after injury. Upon initial evaluation, the individual had C7 tetraplegia with an American Spinal Injury Association (ASIA) Impairment Scale (AIS) grade B of the lower limbs. The strength of finger flexion was grade 2 and finger abduction was grade 1 . The sensory modalities 
were reduced below $\mathrm{T} 2$ and completely disappeared below L1. The bulbocavernosus reflex was preserved. There was impairment of bladder/bowel and autonomic function. The ASIA motor score was 37 points (out of 100 points). A computed tomography (CT) scan showed a completely dislocated C7 and spondylolisthesis with locked C6 spinous process and vertebral arch into the spinal canal (Fig. 1). CT angiography revealed no evidence of vertebral artery injury. We diagnosed the case as CES 5 spondyloptosis of C7. Closed reduction was absolutely invalid due to rigid locking. Thirty minutes after his arrival at our institute, the individual was transferred to the operating room after an initial evaluation and CT scan

The individual was placed in a prone position with the cervical spine in a neutral position using a Mayfield frame after general anaesthesia. Preoperative closed reduction was attempted but failed because of rigid locking of the cervical spine. The posterior elements were exposed by detaching the ligamentous attachments to the spinous processes. We intended to expose the lateral wedge of the C6-7 facet joint, which may hinder cervical reduction. The dislocated vertebrae could not be released using simple traction due to rigid locking. Therefore, the cranial portion of the $\mathrm{C} 7$ vertebral arch and the ligamentum flavum were resected, and then the cervical spine was set in a more kyphotic position by adjusting the Mayfield frame. Simultaneously, two nerve retractors were inserted into the bilateral joint space, and the cervical dislocation was reduced using a protective circular movement based on the lever rule (Fig. 2a, b). Pedicle screws were inserted bilaterally to the sides of C6 and T1 and the left side of $\mathrm{C} 7$, and lateral mass screws were inserted into the bilateral sides of the $\mathrm{C} 5$ vertebra. Titanium rods were inserted by slightly lifting the $\mathrm{C} 6$ vertebra to the dorsal side, and an autologous bone graft was implanted (Fig. 2c). The fascia and subcutaneous tissue were closed using absorbable suture. The total intraoperative blood loss was $30 \mathrm{ml}$.

Two days after surgery, the individual regained the ability to move his lower limbs. Six months after surgery, he had made significant neurological recovery of his lower limbs (AIS grade D). He improved to 89 points on the ASIA
Fig. 1 Preoperative computed tomography (CT) scans of the cervical spine. Three-

dimensional reconstruction (a), sagittal (b), and axial (c) CT scans showed $\mathrm{C} 7$ spondyloptosis locked C6 spinous process and vertebral arch into the spinal canal. The spinous processes of C5 and C6 had been fractured. The C2-7 lordotic angle was $37^{\circ}$.
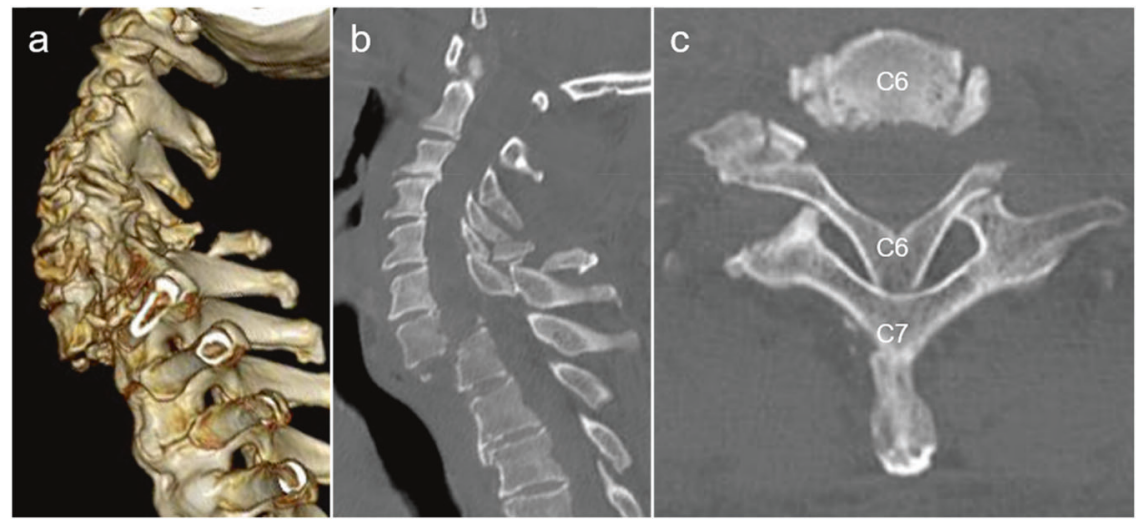
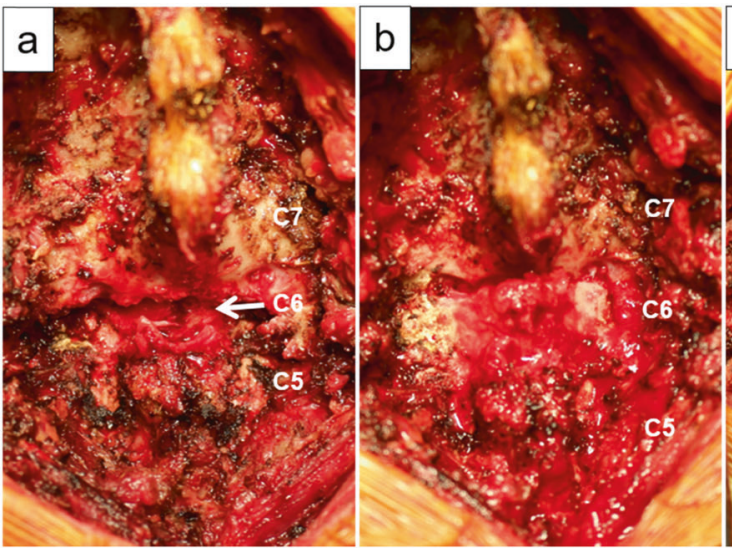

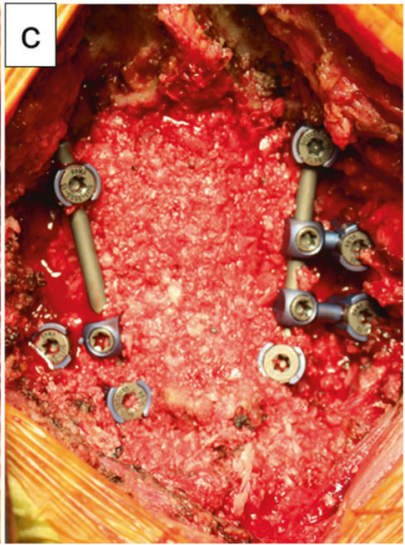

Fig. 2 Intraoperative photographs. a Photograph of the intraoperative cervical spine before reduction. The C6 spinous process and vertebral arch could not be confirmed clearly because they were behind the C7 vertebral arch (white arrow). b Photograph of the intraoperative cervical spine after reduction. The C6 spinous process, vertebral arch, and bilateral facet joint were released from rigid locking. c Photograph of the intraoperative cervical spine after instrumentation and autologous bone grafting. 
Fig. 3 Postoperative images. Postoperative lateral radiograph (a) and computed tomography (CT) scans (b) of the cervical spine. Stabilisation was performed from the $\mathrm{C} 5$ to $\mathrm{T} 1$ vertebrae with a pedicle screw and lateral mass screws.
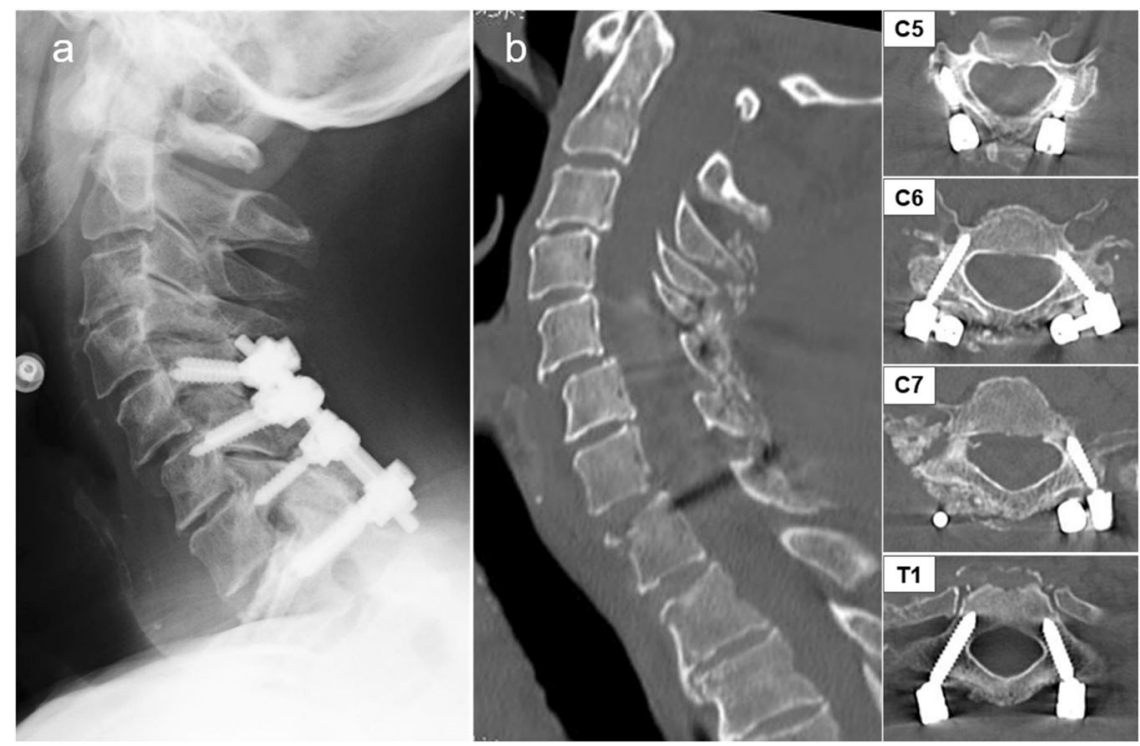

motor score and acquired a stable gait using a walker. Radiographic examinations showed bone union of the $\mathrm{C} 7$ vertebra, and a posterior fusion mass had formed at the C6-7 vertebrae (Fig. 3).

\section{Case 2}

A 73-year-old man with extreme neck pain after falling from a height of $1 \mathrm{~m}$ was transferred to our institute $5 \mathrm{~h}$ after his injury. The individual had grade 4 weakness of left finger abduction and bilateral reduction of sensory modalities between C8 and T2 (AIS grade D). There was no impairment of bladder/bowel and autonomic function. His ASIA motor score was 99 points. A CT scan showed a complete C7-T1 spondylolisthesis with locked C6 spinous process and vertebral arch into the spinal canal (Fig. 4). CT angiography revealed no evidence of vertebral artery injury. Closed reduction was difficult due to rigid locking, and he was transferred to the operating room after an initial evaluation and CT scan.

Preoperative closed reduction was achieved with mild traction with the individual in a prone position and the cervical spine in a neutral position using a Mayfield frame after general anaesthesia. After reduction, spinal fusion surgery from $\mathrm{C} 5$ to $\mathrm{T} 1$ vertebrae was conducted with a pedicle screw and lateral mass screws, and an autologous bone graft was implanted. The total intraoperative blood loss was $330 \mathrm{ml}$. Six months after surgery, he had no neurological deficits of his extremities and his ASIA motor score was 100 point (AIS grade E). Radiographic examinations showed the formation of a posterior fusion mass at the C6-7 vertebrae (Fig. 5).
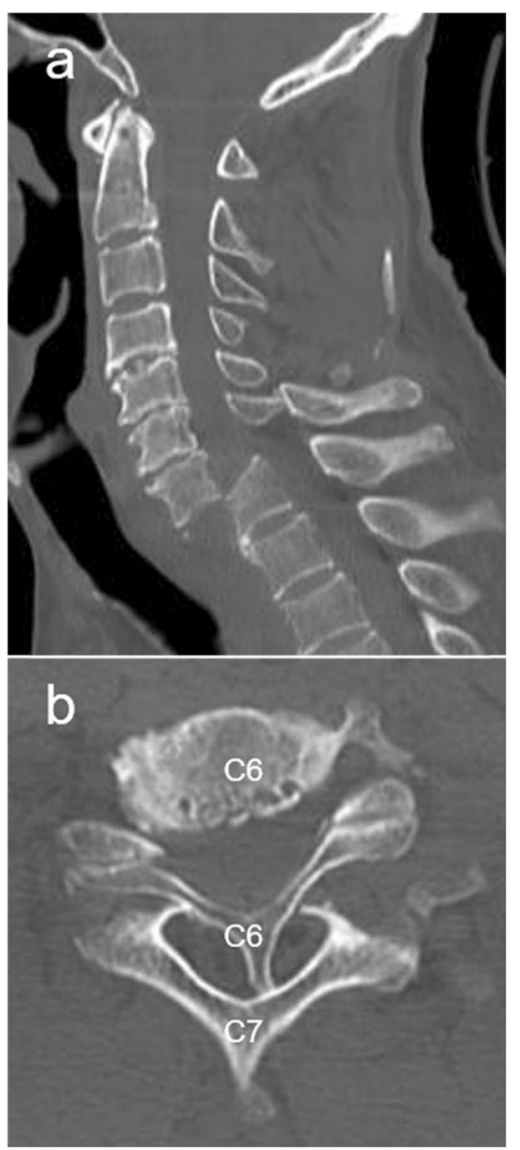

Fig. 4 Preoperative images of case 2. Preoperative sagittal (a) and axial (b) computed tomography (CT) scans of the cervical spine. The $\mathrm{C} 7$ vertebra was completely dislocated and the C6 spinous process was locked by the $\mathrm{C} 7$ vertebral arch. The spinous process of $\mathrm{C} 6 \mathrm{had}$ been fractured. The $\mathrm{C} 2-7$ lordotic angle was $18^{\circ}$. 


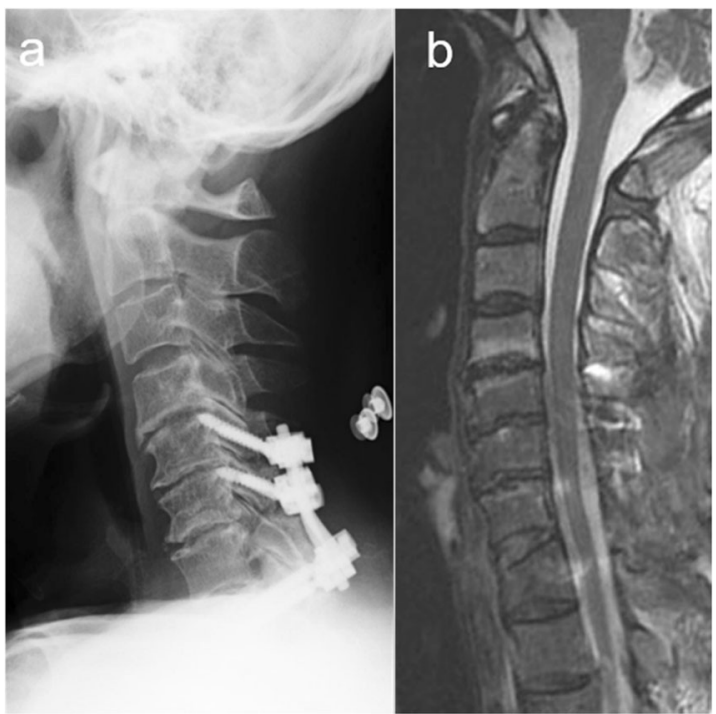

Fig. 5 Postoperative images of case 2. Postoperative lateral radiograph (a) and magnetic resonance image (MRI) (b) of the cervical spine. The patient underwent spinal fusion surgery from C5 to T1 vertebra with a pedicle screw and lateral mass screws.

\section{Discussion}

Traumatic cervical spondyloptosis, in which the destroyed vertebral body is completely dislocated to the anterior side of the subjacent vertebral body, is an extremely severe injury [1-4]. Most individuals develop a complete or nearly complete spinal cord injury [6]. Traumatic cervical spondyloptosis is not uncommon, although cases involving locked spinous process and vertebral arch into the spinal canal are extremely rare and have not been reported previously.

Traumatic cervical spondyloptosis often represents CES 5 of Allen's classification of cervical spine injuries [3, 4, 7-9]. A CES 5 injury consists of anterior displacement of the full vertebral body width with bilateral vertebral arch fracture, and usually the posterior elements of the fractured vertebra do not displace [5]. The spinous process of the suprajacent vertebra generally displaces anteriorly coincident with suprajacent vertebral body displacement. However, in the cases presented here, the C6 spinous process and vertebral arch were displaced under the C7 vertebral arch, which made a closed reduction impossible due to rigid locking. This type of injury, which we termed 'locked spondyloptosis', is the most severe form of traumatic spondylolisthesis.

The success or failure of closed reduction may depend on the degree of cervical lordosis. The cervical spine in both of our cases was rigidly fixed due to the locked spondyloptosis, although we could achieve closed reduction in the second case despite failure of closed reduction in the first case. The two cases differed in the degree of cervical lordosis. Specifically, in the first case, the cervical lordosis was high, whereas in the second case, it was low. Generally, traction in the neck flexion position is effective for reduction of cervical dislocation in a compressive-extension injury [10]. Therefore, the second case with a lower cervical lordosis was closer to the flexion state and therefore was possibly advantageous for closed reduction.

Traumatic cervical spondyloptosis is an extremely unstable condition with destruction of all three spinal columns. Stabilisation surgeries for traumatic cervical spondyloptosis have been reported in the literature [9, 11-13], and an anterior-posterior combined fusion is frequently performed $[9,11,13]$. However, stabilisation using instrumentation with a posterior approach alone is available by ensuring reliable anchors, such as pedicle screws or multiple lateral mass screws [14-16]. Surgery with a posterior approach alone may be feasible and is an ideal option for the treatment of traumatic locked spondyloptosis for which early open reduction and stabilisation are necessary.

These two rare cases presented the most severe traumatic cervical spondyloptosis, namely, traumatic locked spondyloptosis. To the best of our knowledge, this is the first report discussing the management of traumatic locked spondyloptosis. Urgent open reduction is required for locked spondyloptosis when closed reduction is invalid due to rigid locking of the cervical spine.

\section{Compliance with ethical standards}

Conflict of interest The authors declare that they have no conflict of interest.

Publisher's note Springer Nature remains neutral with regard to jurisdictional claims in published maps and institutional affiliations.

\section{References}

1. Gasco J, Dilorenzo DJ, Patterson JT. C4-C5 post-traumatic spondyloptosis with in situ fusion: systematic literature review and case report. Spine (Philos Pa 1976). 2013;38:E621-5.

2. Modi JV, Soman SM, Dalal S. Traumatic cervical spondyloptosis of the subaxial cervical spine: a case series with a literature review and a new classification. Asian Spine J. 2016;10:1058-64.

3. Padwal A, Shukla D, Bhat DI, Somanna S, Devi BI. Posttraumatic cervical spondyloptosis: a rare entity with multiple management options. J Clin Neurosci. 2016;28:61-6.

4. Srivastava SK, Agrawal KM, Sharma AK, Agrawal MD, Bhosale SK, Renganathan SR. C3-C4 spondyloptosis without neurological deficit-a case report. Spine J. 2010;10:e16-20.

5. Allen BL Jr, Ferguson RL, Lehmann TR, O'Brien RP. A mechanistic classification of closed, indirect fractures and dislocations of the lower cervical spine. Spine (Philos Pa 1976). 1982;7:1-27.

6. Ahmad FU, Wang MY. The case for aggressive management in traumatic high-grade cervical dislocations. World Neurosurg. 2014;82:1055-7.

7. Dahdaleh NS, Dlouhy BJ, Greenlee JD, Smoker WR, Hitchon PW. An algorithm for the management of posttraumatic cervical spondyloptosis. J Clin Neurosci. 2013;20:951-7. 
8. Dhall SS, Sribnick EA. Significant neurologic recovery after traumatic cervical spondyloptosis and spinal cord injury. Spine J. 2014;14:194-5.

9. Tumialan LM, Dadashev V, Laborde DV, Gupta SK. Management of traumatic cervical spondyloptosis in a neurologically intact patient: case report. Spine (Philos Pa 1976). 2009;34:E703-8.

10. Herkowitz HN, Garfin SR, Eismont FJ, Bell GR, Balderston RA. Rothman-Simeone, The Spine E-Book: Expert Consult. Elsevier Health Sciences, Philadelphia, 2011.

11. Menku A, Kurtsoy A, Tucer B, Oktem IS, Akdemir H. The surgical management of traumatic C6-C7 spondyloptosis in a patient without neurological deficits. Minim Invasive Neurosurg. 2004;47:242-4.

12. Shah KC, Rajshekhar V. Successful management of posttraumatic C7-T1 spondyloptosis with uninstrumented ventral surgery. Surg Neurol. 2004;62:431-4.
13. Ramieri A, Domenicucci M, Cellocco P, Lenzi J, Dugoni DE, Costanzo G. Traumatic spondylolisthesis and spondyloptosis of the subaxial cervical spine without neurological deficits: closed realignment, surgical options and literature review. Eur Spine J. 2014;23:658-63.

14. Abumi K, Itoh H, Taneichi H, Kaneda K. Transpedicular screw fixation for traumatic lesions of the middle and lower cervical spine: description of the techniques and preliminary report. J Spinal Disord. 1994;7:19-28.

15. Bozkus H, Ames CP, Chamberlain RH, Nottmeier EW, Sonntag VK, Papadopoulos SM, et al. Biomechanical analysis of rigid stabilization techniques for three-column injury in the lower cervical spine. Spine (Philos Pa 1976). 2005;30:915-22.

16. Abumi K, Ito M, Sudo H. Reconstruction of the subaxial cervical spine using pedicle screw instrumentation. Spine (Philos Pa 1976). 2012;37:E349-56. 